\title{
ARTICLES \\ Responsible Innovation for Life: Five Challenges Agriculture Offers for Responsible Innovation in Agriculture and Food, and the Necessity of an Ethics of Innovation
}

\section{Bart Gremmen $^{1}$ D $\cdot$ Vincent Blok $^{1} \cdot$ Bernice Bovenkerk $^{1}$}

Accepted: 5 October 2019 / Published online: 18 October 2019

(c) The Author(s) 2019

\begin{abstract}
In this special issue we will investigate, from the perspective of agricultural ethics (e.g. animal welfare, agricultural and food ethics, environmental ethics etc.) the potential to develop a Responsible Research and Innovation (RRI) approach to agriculture, and the limitations to such an enterprise. RRI is an emerging field in the European research and innovation (R\&I) policy context that aims to balance economic, socio-cultural and environmental aspects in innovation processes. Because technological innovations can contribute significantly to the solution of societal challenges like climate change or food security, but can also have negative societal consequences, it is assumed that social and ethical aspects should be considered during the R\&I process. For this reason, the emerging concept of RRI calls for ethical reflection on the nature, scope and applicability of responsibility and innovation in innovation practices in general, and the way social-ethical issues can be applied and addressed in agriculture.
\end{abstract}

Keywords Responsible innovation · Citizen inclusion · Drones · Gene editing · Ecological management

The global consumption of agricultural products is expected to rise (FAO 2012). However, agriculture in Western Europe faces a variety of problems, regarding for instance the reduced welfare of animals in housing systems in the 1970s, the use of pesticides in the early 80 s, susceptibility to infectious diseases in the 90 s, and at the beginning of the twenty-first century erosion and the high use of antibiotics and vaccines. These problems will decrease the amount of available future agricultural

Bart Gremmen

bart.gremmen@wur.nl

1 Department of Philosophy, Wageningen University and Research, Hollandseweg 1, $6706 \mathrm{KN}$ Wageningen, The Netherlands 
products. Could agricultural research solve most of these problems by a series of innovations?

Incremental innovation has always been at the center of agriculture. Farmers have from the start tried to improve the use of plants and animals for human consumption. By using trial and error breeding processes they slowly domesticated crop plants and animals. This process of thousands of years of domestication has led to an enormous extension of cultivated fields, with only a few kinds of uniform crop plants that are produced in monocultures, and the use of mainly five species of animals in animal livestock farming. Next to incremental innovation, in the last few hundred years, agricultural research has led to many radical innovations in agriculture. These innovations, ranging from mechanization to hybrid cultivars and from the Green Revolution to modern biotechnology, have shaped conventional agriculture on a global scale. However, in recent years especially these radical innovations have led to much criticism from various kinds of non-governmental organizations and policy makers. The main points of criticism are centered on pesticide-, and fertilizer use, soil degradation and use of genetically modified plants. There is also a growing awareness among citizens about these issues and the joined efforts of animal rights activists and environmental groups. As a consequence, agriculture has almost lost its licenseto-produce from society. In order to regain this license to produce, various initiatives have been taken to alter production processes. For instance, critics argue that individual plants and animals are not just instruments for profit driven enterprises, but also have an intrinsic value that commands respect (Bekoff et al. 1992). Conventional farmers are thus urged to reorganize their practices in a responsible way and no longer consider plants and animals as mere instruments or objects.

In this special issue we will investigate, from the perspective of agricultural ethics (e.g. animal welfare, agricultural and food ethics, environmental ethics etc.) the potential to develop a Responsible Research and Innovation (RRI) approach to agriculture, and the limitations to such an enterprise. Responsible Research and Innovation (RRI) is an emerging field in the European research and innovation (R\&I) policy context that aims to balance economic, socio-cultural and environmental aspects in innovation processes (European Commission 2011). Because technological innovations can contribute significantly to the solution of societal challenges like climate change or food security, but can also have negative societal consequences, it is assumed that social and ethical aspects should be considered during the R\&I process. For this reason, the emerging concept of RRI calls for ethical reflection on the nature, scope and applicability of responsibility and innovation in innovation practices in general, and the way social-ethical issues can be applied and addressed in agriculture. The concept of RRI expresses the ambition to address societal needs, next to the more traditional objectives of R\&I like economic growth, profit maximization, competitive advantage etc. (cf. Blok and Lemmens 2015a).

In the context of agriculture and food, RRI offers opportunities because it may provide a new impulse to strengthen animal-, agricultural-, and food ethics. An example is reflection on the killing of day old male chicks (Bruijnis et al. 2015; Gremmen et al. 2018). Compared to a 'classical' innovation trajectory, responsible innovation aims at some kind of stakeholder involvement at an early stage (Blok and Lemmens 2015b; Blok 2019). In this way the societal values of the 
stakeholders can be discussed. An example in agriculture is the so-called reflexive interactive design method (Bos et al. 2009).

Although RRI has a lot of potential, it also provides particular context-specific challenges. RRI in agriculture and food entails more than the application of dimensions of RRI to artefacts. Agriculture can be defined as the technical transformation of living material into plant and animal products, in which life processes play an essential role. Agricultural products enter our body, which is why farming, as a kind of interface, provides a sharp distinction between the (outside) world of nature and the (inside) world of humans. In this way we are included in larger systems and part of many cycles. The products in farming are organic, while in all other technical practices the products are inorganic. Without a doubt, agriculture is the most crucial human activity on earth. Farmers don't just take care of plants and animals, but do this to let us survive as human species. The first challenge agriculture offers RRI is that we cannot immediately abandon irresponsible agricultural processes and products in the way we can abandon irresponsible other technological processes and products. Without agriculture we would be forced to collect the limited food that is available in nature again. As a result, the number of people on the earth would decrease drastically. This means that agriculture is necessary for the survival of humanity, while we can do without most other technical practices. Although we can survive without some agricultural products, like meat, our culture is entirely focused on plant and meat consumption. In this way the plants and animals "take care" of us. Agriculture is the basis of our culture whereby, by intervening in the natural life of plants and animals, we are only gradually able to create a domesticated nature.

But what do we mean by the term "nature"? Much has been written about the concepts of "nature" and "naturalness". This varies from romantic rural associations and ideas about the goodness and pureness of nature to a reservoir for human use, from a biological or ecological system that can be discovered to a self-healing whole, to name just a few meanings. For example, in recent social debates about pig farming and the use of mega stalls, the concept of "naturalness" seems to emerge as a certain kind of argument referring to very different contexts: the environment, animal integrity, technology, quality of life, etc. Nature is not only a certain category that expresses the separation between man and the surrounding natural world, but it also functions as a normative standard (if something is unnatural, it is not good) that guides change and sets the limits of our control over it (van Haperen et al. 2012). This leads to the second challenge agriculture offers for RRI: in agricultural innovations, intrinsic normative standards seem to be provided and used, contrary to other innovations which provide no intrinsic normative standards at the start of the innovative process and call for extrinsic normative standards like in value sensitive design. There is a symbiosis between people and domestic plants and animals in farming, as a result of which we can regard farming as a kind of "second" nature. Animals in nature are, to a certain extent, autonomous: they do not need us to survive. We have made domestic plants and animals dependent on us by usually intervening in their life processes, especially their reproduction. The farmer is the one who takes care of living things at the farm, also the one who always, with the help of technology, makes a selection from everything that lives. This turns the farmer, 
also in his or her modern role as manager, from someone who reproduces life into someone who produces life.

However, innovations leading to irreversible actions, such as the extinction of certain natural species by genetic modification, or the destruction of all the seeds of a cultivar, are very risky because interrelated cycles that continue to make the future of farming possible could be interrupted. This means that the artefacts resulting from non-agricultural innovations may have certain one-dimensional risks, while in agriculture often multi-dimensional risks may occur because of the interrelatedness of species in (agricultural) ecosystems. Taking care of these multi-dimensional risks is the third challenge agriculture offers RRI. For example the invention of genetic modification in 1973 has been important for agriculture because it allows direct intervention in the genetic basis of the reproduction of plants and animals. This technology is also called transformational technology because it not only enables the manipulation of life at the most fundamental, molecular level, but above all because it fundamentally changes the conditions of possibility of the transformation of life itself. The trial and error methods of the farmers are replaced by the targeted approach of scientists, therefore giving the concept of "domestication" a completely new meaning.

In farming, plants and animals are not only a production goal but also a means of production. An important difference between innovation of artefacts and animals and plants is that until now we have not designed the products in farming ourselves through a blueprint on the drawing board. There is talk of "designing" via domestication and targeted breeding, but that is highly dependent on the biodiversity present and the natural path dependencies of the genetic material. But at this moment plants and animals in farming are not designed by us and they function relatively autonomously as means of production. In farming, the products produce themselves. They have, however, been transformed from natural organisms into domesticated organisms. This leads to a fourth challenge for RRI which normally reflects only on artefacts. Contrary to artefacts, organisms and agro-ecosystems have a double ethical status: they are both subjects and objects of the innovation process. In farming there is no distinction between production process and product: through the process of growth, the products produce themselves, in contrast to practices in which people or machines make the products. In farming, people only provide the preconditions.

From a more general ethical point of view one could argue that RRI is somewhat ambiguous. On the one hand it promises a clear ethical agenda (Von Schomberg 2019; Van de Poel and Robaey 2017), on the other hand it contains the risk that ethics (responsibility) is reduced to procedural categories such as deliberation and responsiveness (Stilgoe et al. 2013). In contexts with living organisms, like agriculture, this reduction is counterproductive. In order to do justice to the actual assignment of RRI, ethics should, in these contexts, belong to the core of RRI. In our view this is the fifth and main challenge agriculture offers RRI. The ethically unique status of farming means that the mainstream approach to RRI cannot be simply applied to the agricultural and food context. It requires strengthening the ethical agenda of RRI in order to enable contextualization of RRI in the domain of agriculture. This means that sectoral contextualisation of RRI does not only imply the broadening the scope of applicability of RRI but that the focus of the mainstream concept of RRI 
has to shift towards ethics of innovation in order to become applicable in the context of agriculture and food. The reduction of ethics in RRI to deliberation and responsiveness may explain why the adoption of RRI is still in its infancy in the agriculture and food sector.

In order to take more fully advantage of the potential of RRI for animal-, agriculture- and food ethics, this special issue focusses on the development of an agriculture and food approach to RRI that is sensitive to these specific contextual factors. We propose the following research agenda for RRI in the agriculture and food sector:

- Is the concept of RRI as such applicable in the context of agriculture?

- The nature of responsibility in light of the specific context of agricultural innovations.

- How to integrate the double responsibility towards organisms and eco-systems as both subject and object of innovation in RRI approaches in the agriculture and food sector?

- What are the characteristics of RRI in agriculture and food?

- How can the application of the RRI framework help to better embed agricultural and food technologies in society?

- How can the RRI framework be applied in the assessment of controversial emerging technologies in the agricultural sector (Precision Livestock Farming, genetic modification, CRISPR-Cas etc.)?

- How can the RRI concept be applied to improve animal welfare?

Together the contributors to the special issue elaborate on most of the questions of this ethical agenda of RRI in agriculture. They explore the opportunities, advantages and disadvantages of the emerging concept of RRI in the field of animal-, agriculture- and food ethics. Our aim is to use the papers as the start of a strategic discussion with all relevant stakeholders in order to apply RRI to agriculture.

\section{Overview of the Special Issue}

Agricultural production today faces several challenges: an increasing world population has to be fed, but at the same time the environment and animal welfare need to be protected. Globalisation has led to increasing international competition, while the agricultural sector is also under heavy economic pressure as a result of regulation. These conditions can perhaps only be met by innovation, be it on the technological or on the systems-level. Innovation itself comes with ethical, institutional, and legislative challenges, however. As Frankelius, Norman and Johansen show in their analysis of the introduction of unmanned aerial systems (or drones), legislative institutions can inhibit responsible innovation. How to balance the benefits of using drones with guaranteeing physical safety? How to guarantee that individual privacy is not infringed? Their study shows that different ethical perspectives can collide with each other, but that ethical analyses of the use of drones have tended to emphasize its negative implications, such as safety, 
privacy, and integrity concerns, dehumanization and potential misuse, thereby neglecting potential opportunities and benefits of the technology, in particular its potential to address food security and environmental sustainability. Devitt, Baxter and Hamilton's contribution also discusses the use of drones, or remotely piloted aircraft systems, in particular as a form of biosecurity surveillance, in order to keep agricultural systems free of pests. Their focus is not in the first place on institutional or regulatory challenges, but on the inclusion of a diverse group of stakeholders. They discuss the tension between on the one hand food security by keeping agriculture pest-free and on the other hand potentially resulting privacy violations and reduced farmer autonomy. They propose an ethical framework for biosurveillance that is based on RRI and that should lead to just and inclusive use of these activities, co-created by diverse stakeholders.

In their contribution, Eastwood, Klerkx, Ayre and Dela Rue, also call for inclusion, but in this case of citizens and consumers. They focus on smart farming methods in the dairy industry by analysing a case study of smart dairying research and development in New Zealand and in particular the extent to which RRI principles were applied. The application of RRI left something to be desired in this case study. The innovation targeted technology development and on-farm use of technologies and largely neglected socio-ethical implications of smart farming. The authors call for stronger leadership by government and the dairy sector in order to create more readiness to apply RRI principles.

Ann and Donald Bruce in their contribution assess the four core RRI principles of anticipation, reflexivity, inclusion and responsiveness in the case of genome editing of livestock, in particular focussing on hornless cattle and disease resistant pigs. Like Eastwood et al. they focus on including citizens in innovation processes, but they also point out obstacles. Firstly, RRI calls for a slow reflective pace, whereas research and development of genome editing - spurred on by the many potential uses of the CRISPR method-are going full steam ahead. Secondly, attempts to engage the public tend to reach only a small number of people. Thirdly, a knowledge gap exists, as consumers are generally not aware of how the food they consume is produced. They often do not recognize the potential animal welfare improvements that genome editing can bring, although research suggests that applications of genome editing for reducing antibiotic use, greenhouse gas emissions and zoonotic diseases are regarded more positively. Moreover, citizens tend to ask questions about ownership of new technologies. Not taking these public views seriously can backfire and therefore, the authors are in favour of a more inclusive and reflective, and therefore a slower, process. They argue that this can best be achieved not during the phase of $\mathrm{R} \& \mathrm{D}$, but during the slower phase of taking a product from the lab to the market. Therefore, they suggest we put the 'I' back into RRI.

The final contribution, by Stojanovic, focuses on innovation at the systems-level. The paper investigates the novel integrative approach of ecological management, which aims to link ecological and economic models to ecological and economic behaviour. This has implications for innovative (agricultural) systems such as agroecology and circular economy. The author argues that ecological problems are closely tied to a lagging education system, raising the question of what role education has to play in Responsible Research and Innovation. 
Open Access This article is distributed under the terms of the Creative Commons Attribution 4.0 International License (http://creativecommons.org/licenses/by/4.0/), which permits unrestricted use, distribution, and reproduction in any medium, provided you give appropriate credit to the original author(s) and the source, provide a link to the Creative Commons license, and indicate if changes were made.

\section{References}

Bekoff, M., Gruen, L., Townsend, S. E., \& Rollin, B. E. (1992). Animals in science: Some areas revisited. Animal Behaviour, 44(3), 473-484.

Blok, V. (2019). From participation to interruption: Toward an ethics of stakeholder engagement, participation and partnership in corporate social responsibility and responsible innovation. In R. Von Schomberg (Ed.), International handbook on responsible innovation. A global resource (pp. 243257). Cheltenham: Edward Elgar.

Blok, V., \& Lemmens, P. (2015a). Critical reflections on the concept of responsible innovation. In E. J. Koops, J. van den Hooven, H. A. Romijn, T. E. Swierstra, \& I. Oosterlaken (Eds.), Responsible innovation: Issues in conceptualization, governance and implementation. Dordrecht: Springer.

Blok, V., \& Lemmens. P. (2015b). Critical reflections on the concept of responsible innovation. In: B.J. Koops, I. Oosterlaken, H. Romijn, T. Swierstra, \& J. van den Hoven (Eds.), Responsible innovation 2. Concepts, Approaches, and Applications. Dordrecht: Springer

Bos, A. P., Groot Koerkamp, P. W. G., Gosselink, J. M. J., \& Bokma, S. (2009). Reflexive interactive design and its application in a project on sustainable dairy husbandry systems. Outlook on Agriculture, 38(2), 137-145.

Bruijnis, M. R. N., Blok, V., Stassen, E. N., \& Gremmen, H. G. J. (2015). Moral 'Lock-In' in responsible innovation: The ethical and social aspects of killing day-old chicks and its alternatives. Journal of Agricultural and Environmental Ethics. https://doi.org/10.1007/s10806-015-9566-7.

European Commission. (2011). Horizon 2020-The framework programme for research and innovation. Brussels: European Commission.

FAO. (2012). The state of food insecurity in the world. Rome: FAO.

Gremmen, B., Bruijnis, M. R. N., Blok, V., \& Stassen, E. N. (2018). A public survey on handling male chicks in the Dutch egg sector. Journal of Agricultural and Environmental Ethics. https://doi. org/10.1007/s10806-018-9712-0.

Stilgoe, J., Owen, R., \& Macnaghten, P. (2013). Developing a framework for responsible innovation. Research Policy, 42(9), 1568-1580.

Van de Poel, I., \& Robaey, Z. (2017). Safe-by-design: From safety to responsibility. NanoEthics, 11(3), 297-306.

van Haperen, P. F., Gremmen, H. G. J., \& Jacobs, J. G. M. (2012). Reconstruction of the ethnical debate on naturalness in discussion about plant-biotechnology. Journal of Agricultural and Environmental Ethics, 25(6), 797-812.

Von Schomberg, R. (2019). International handbook on responsible innovation: A global resource. Cheltenham: Edward Elgar.

Publisher's Note Springer Nature remains neutral with regard to jurisdictional claims in published maps and institutional affiliations. 